\title{
Performance of age-adjusted D-dimer values for predicting DVT before the knee and hip arthroplasty
}

\author{
Jian Xiang $W^{1,2}$, Jiang Hui Qing ${ }^{2}$, Yao Yao ${ }^{2}$, Dong Yang $C^{2}{ }^{2}$ and Qing Jiang ${ }^{2^{*}}$ (D)
}

\begin{abstract}
Purpose: To compare the specificity and sensitivity of preoperative D-dimer and age-adjusted D-dimer value for predicting the incidence of the DVT preoperatively in total joint arthroplasty (TJA) patients.

Methods: We enrolled 406 patients finally above 50 years old. Everyone had done ultrasonography bedside, and Ddimer concentrations were collected before surgery. The D-dimer and age-adjusted D-dimer cut-off was calculated by multiple logistic regression and receiver operating curve (ROC) analyses.

Results: A total of 39 patients had found asymptomatic deep vein thrombosis (DVT) by ultrasonography. The age (odds ratio $[\mathrm{OR}] 1.067 ; p=0.003$ ) and D-dimer (OR 1.331; $p=0.025)$ were related to the existence of DVT. For conventional D-dimer and age-adjusted D-dimer value, the area under the curves (AUCs) were 0.685 (0.499-0.696) and 0.795 (0.611-0.881), respectively.
\end{abstract}

Conclusion: Compared to traditional D-dimer, age-adjusted D-dimer showed better performance in screening DVT, which was useful clinically.

\section{Introduction}

As is known to all, the total hip arthroplasty (THA) and total knee arthroplasty (TKA) are the effective methods to treat osteoarthritis, but it also can bring some complications, such as infection, pain, and deep vein thrombosis (DVT), which was the most common complication. The incidence of DVT is about $11.9 \%$ in THA and $20.8 \%$ in TKA after surgery [1]. DVT can lead to Pulmonary thromboembolism (PTE), which is the most serious complication after arthrosurgery. There are many researches that focus on the incidence of DVT postoperatively; few studies about preoperative DVT were reported [2]. In clinical work, the removal of

\footnotetext{
* Correspondence: qingj@nju.edu.cn

${ }^{2}$ State Key Laboratory of Pharmaceutical Biotechnology, Department of Sports Medicine and Adult Reconstructive Surgery, Nanjing Drum Tower Hospital, The Affiliated Hospital of Nanjing University Medical School, 321 Zhongshan Road, Nanjing 210008, Jiangsu, P.R. China

Full list of author information is available at the end of the article
}

thrombus preoperatively may need the help of imaging examination such as ultrasound and contrast radiography. However, there are inadequacies in all the above examinations, such as time-consuming, expensive, and labor-consuming, so they are not suitable for large-scale use before operation. If patients have asymptomatic DVT preoperatively, it might cause severe consequence. Thus, a valuable method in detection of DVT or PTE before surgery is necessary.

In clinical work, the prompt diagnosis for thrombosis is the $\mathrm{D}$-dimer test, which can give references to the surgeon [3]. D-dimer is produced by fibrin degradation [4]. The monitoring value of $\mathrm{D}$-dimer is identified according to different methods. The cut-off value of D-dimer is $0.5 \mu \mathrm{g} / \mathrm{ml}$ by latex agglutination turbidimetry (LATEX) in our hospital; however, in the elderly patients, the Ddimer concentrations are higher than the upper limit of normal age [5]. Thus, the value of D-dimer is discounted in the elderly patients [6]. Moreover, the pregnancy, 
infection, inflammation, and cancer also can lead to high $\mathrm{D}$-dimer value. As a result, the only use of D-dimer to predict DVT is not appropriate.

Some studies showed that the use of age-adjusted Ddimer cut-off in the diagnostic strategy for the specificity and sensitivity of deep vein thrombosis is more valuable over 50 years old $[7,8]$. Nybo and Hvas argued that the use of an age-adjusted D-dimer in patients above 50 years of age for ruling out DVT seems as safe as using a standard D-dimer cut-off [9]. Schouten et al. also described that the application of age-adjusted cut-off values to the $\mathrm{D}$-dimer value $(0.01 \times$ age [years] $)$ considerably elevates specificity without altering sensitivity in patients aged $>50$ years with suspected PTE [10].

Therefore, the use of D-dimer concentrations for predicting deep vein thrombosis is controversial [11]. However, the application of age-adjusted cut-off values to the D-dimer value is relatively rare in orthopedics. So, it is valuable for us to research. The method of this study is to compare the specificity and sensitivity of preoperative D-dimer and age-adjusted D-dimer value for predicting the incidence of the DVT preoperatively. Our aim is to explore the feasibility and effectiveness of this method in the field of joint replacement.

\section{Materials and methods}

We enrolled 834 patients, whose age was over 50 years old, who underwent THA or TKA at Affiliate Drum Tower Hospital, Medical School of Nanjing University from September 1, 2015, to April 1, 2017. All patients signed the informed consent. The patients who underwent the pregnancy, infection, and cancer were excluded. In addition, patients were excluded if they had a femoral neck fracture, whose concentration of D-dimer was above normal level. Also, patients with cardiovascular disease and a previous history of thromboembolism were excluded. We removed the patients who had taken anticoagulant or antiplatelet agents previously. In the end, we included 406 patients in our study. All the patients drew blood for collecting the plasma D-dimer level when they were admitted to hospital. The cut-off value of D-dimer was $0.5 \mu \mathrm{g} / \mathrm{ml}$ by latex agglutination turbidimetry (LATEX). We divided patients aged > 50 into 10-year age groups. Renee A Douma made three large cohorts to find the age-adjusted D-dimer value. They plotted the D-dimer cut-off level against age group and performed linear regression analysis to obtain the regression coefficient. They finally proved the multiplication factor for age in the new age-adjusted cut-off value was 0.01 . Thus, the age-adjusted $\mathrm{D}$-dimer value was calculated as age (years) $\times 0.01 \mu \mathrm{g} / \mathrm{mL}$. The plasma Ddimer level, diagnosis, age, sex, and body mass index (BMI) of the patients were recorded preoperatively. All the patients did not take anticoagulant or antiplatelet agents. The surgeries were done by four skilled orthopedic surgeons. The patients accepted the same program of rehabilitation and postoperative management. We observed vein thrombosis 1 day preoperatively. The skilled radiologists performed thrombosis examinations for all patients. The double lower limbs were observed with bedside ultrasound machine with two mode (B-mode and color Doppler ultrasound examination) for the common femoral, popliteal, superficial, and calf veins bilaterally. DVT was diagnosed if (1) the vein could not be compressed and (2) there were abnormal vascular signals in Doppler color ultrasound. Plasma D-dimer normal value was defined as $0.5 \mu \mathrm{g} / \mathrm{ml}$. We wanted to analyze the age-adjusted D-dimer cut-off to predict the DVT event. At the same time, we also analyzed the risk factors for preoperative deep venous thrombosis. We predicted that the age-adjusted D-dimer was a more valuable index for monitoring the DVT before the TKA and THA.

All statistical analyses were from SPSS (version 25; SPSS Inc., Chicago, IL, USA). The qualitative data such as the number of females or males was analyzed by Fisher's exact test. The quantitative data such as body height, body weight, age, body mass index, and D-dimer value was analyzed by unpaired Student's $t$ test. Multiple logistic regression analysis was used to discover the following risk factors related to the presence of DVT: body height, body weight, body mass index (BMI), age, sex, and Ddimer value. Receiver operating characteristic (ROC) curves were produced for age-adjusted D-dimer value and conventional D-dimer value. Also, the area under the curve (AUC) was calculated by ROC curves. In addition, we used the Youden index [12] to determine the cut-off value. We considered $p<0.05$ to be statistically significant.

\section{Results}

In the end, we included 406 patients. There were 118 males and 288 females. The average BMI of all patients was $25.3 \pm 3.9 \mathrm{~kg} / \mathrm{m}^{2}$. The mean age was $65.4 \pm 8.4$ years old. The patients' detailed preoperative basic data and related blood tests are shown in Table 1 .

We divided patients aged $>50$ into 10 -year age groups. We found that the incidence of thrombosis in the group older than 80 years old was higher than that in the other groups, and the difference was statistically significant. In addition, we compared conventional D-dimer and ageadjusted D-dimer cut-off by age group; more patients below cutoff values could be found in all age groups using age-adjusted D-dimer cut-off values. The specific information is shown in Table 2.

A total of 39 patients had found deep vein thrombosis of the lower extremities, all of which were asymptomatic. The incidence of thrombosis was $9.6 \%$. Two of them 
Table 1 Patients' characteristics

\begin{tabular}{lllll}
\hline & Total patients & Patients with DVT & Patients without DVT & $\boldsymbol{p}$ value \\
\hline Patient $(n)$ & 406 & 39 & 367 & - \\
Sex (male/female) & $118 / 288$ & $9 / 30$ & $109 / 258$ & 0.386 \\
Age (years) & $65.4 \pm 8.4$ & $69.8 \pm 6.9$ & $65.0 \pm 8.5$ & $<0.001$ \\
Body weight $(\mathrm{kg})$ & $66.0 \pm 10.8$ & $67.5 \pm 11.6$ & $161.5 \pm 7.0$ & 0.316 \\
Body height $(\mathrm{cm})$ & $161.4 \pm 7.0$ & $160.5 \pm 6.3$ & $25.0 \pm 3.9$ & 0.307 \\
BMl $\left(\mathrm{kg} / \mathrm{m}^{2}\right)$ & $25.3 \pm 3.9$ & $27.2 \pm 3.2$ & $0.66(0.32,0.98)$ & 0.201 \\
Preoperative D-dimer $(\mathrm{\mu g} / \mathrm{ml})$ & $0.45(0.25,0.84)$ & $0.91(0.61,1.12)$ & $28.1 \pm 3.7$ & 0.045 \\
APTT (s) & $28.1 \pm 3.8$ & $28.1 \pm 4.6$ & $12.0 \pm 0.9$ & 0.968 \\
PT (s) & $12.0 \pm 0.9$ & $12.0 \pm 0.8$ & $1.04 \pm 0.08$ & 0.865 \\
INR & $1.04 \pm 0.08$ & $1.04 \pm 0.07$ & $208.2 \pm 62.2$ & 0.927 \\
Platelet count $\left(10^{9} / \mathrm{L}\right)$ & $206.9 \pm 61.7$ & $194.8 \pm 55.8$ & $129.0 \pm 18.9$ & 0.193 \\
Hb (g/L) & $128.7 \pm 18.4$ & $126.1 \pm 12.0$ & 0.091 \\
\hline DVT
\end{tabular}

DVT deep vein thrombosis, BMI body mass index, APTT activated partial thromboplastin time, PT prothrombin time, INR international normalized ratio, $\mathrm{Hb}$ hemoglobin

were iliac vein thrombosis, and the rest were intramuscular venous thrombosis. The mean age of patients with thrombosis was $69.8 \pm 6.9$ years, and the mean age of patients without thrombosis was $65.0 \pm 8.5$ years, with a statistically significant difference $(p<0.001)$. For preoperative D-dimer levels, patients with thrombosis had an average of $0.91(0.61,1.12) \mathrm{mg} / \mathrm{mL}$, compared with $0.66(0.32,0.98) \mathrm{mg} / \mathrm{mL}$ for patients without thrombosis, and there was a statistically significant difference $(p=$ 0.045).

By multivariate logistic regression analysis, when age, gender, BMI, body weight, and preoperative D-dimer levels were independent variables, we found that only age and D-dimer levels were associated with the development of deep vein thrombosis (Table 3).

With regard to preoperative D-dimer, a total of 180 patients had a D-dimer concentration greater than $0.5 \mu \mathrm{g} / \mathrm{mL}$, of which only 18 had thrombosis. With the use of age-adjusted D-dimer, there were only 33 patients' D-dimer concentration above the age-adjusted D-dimer reference value. In addition, we plotted the ROC curve for the age-adjusted D-dimer and the traditional D- dimer (Fig. 1). The area under the curve was 0.795 and 0.685 , respectively (Table 4 ). For DVT observations, the traditional D-dimer had a cut-off value of 0.845 with a sensitivity of $41 \%$ and a specificity of 0.658 (Table 4 ). The cut-off value of age-adjusted D-dimer cut-off value was 0.655 , which caused a sensitivity of 0.769 and a specificity of 0.768 (Table 4).

\section{Discussion}

Patients with joint replacement were generally older. If the traditional $\mathrm{D}$-dimer value was used as a preoperative diagnosis method, the false positive patient would be greatly increased [5].

We used both age-adjusted D-dimer and traditional $\mathrm{D}$-dimer values to detect the effectiveness of deep vein thrombosis. The area under the ROC curve was 0.795 and 0.685 , respectively, and the age-adjusted D-dimer had a better predictive value in predicting deep vein thrombosis. In addition, we found that the sensitivity of the age-adjusted D-dimer cut-off value (76.8\%) was significantly improved compared to the traditional method (41\%), while the specificity, age-adjusted D-dimer cut-off

Table 2 Comparison of different cut-off values stratified by age group

\begin{tabular}{|c|c|c|c|c|c|}
\hline & \multirow[b]{2}{*}{ Total patients } & \multicolumn{4}{|c|}{ Age range (years) } \\
\hline & & $50-59$ & $60-69$ & $70-79$ & $>80$ \\
\hline No (\%) of patients & 406 & $103(25.4)$ & $182(44.8)$ & $99(24.4)$ & $22(5.4)$ \\
\hline Median age (years) & 63.3 & 54.7 & 64.9 & 73.5 & 83.3 \\
\hline No (\%) of patients with DVT & 39 & $3(2.9)$ & $16(8.8)$ & $16(16.2)$ & $4(18.2)$ \\
\hline \multicolumn{6}{|l|}{ Conventional cut-off value } \\
\hline No (\%) of patients below cut-off value & $226(55.7)$ & $73(70.9)$ & $99(54.4)$ & $46(46.5)$ & $8(36.4)$ \\
\hline \multicolumn{6}{|l|}{ Age-adjusted cut-off value } \\
\hline No (\%) of patients below cut-off value & 269 & $78(75.7)$ & $118(64.8)$ & $60(60.6)$ & $12(54.5)$ \\
\hline
\end{tabular}


Table 3 Multiple logistic regression analysis for preoperative deep vein thrombosis

\begin{tabular}{llll}
\hline & OR & $\mathbf{9 5 \% ~ C l}$ & $\boldsymbol{p}$ value \\
\hline Age (year) & 1.067 & $1.023-1.113$ & 0.003 \\
Sex (female) & 0.615 & $0.208-1.814$ & 0.378 \\
Body height $(\mathrm{cm})$ & 0.977 & $0.910-1.049$ & 0.52 \\
Body weight $(\mathrm{kg})$ & 1.022 & $0.988-1.057$ & 0.209 \\
D-dimer $(\mu \mathrm{g} / \mathrm{ml})$ & 1.331 & $1.032-1.716$ & 0.027 \\
\hline
\end{tabular}

Cl confidence interval, $O R$ odds ratio

value $(76.9 \%)$ was also slightly better than the traditional D-dimer (65.8\%).

The basic D-dimer value increased with age. Harper et al. found that D-dimer concentration increased with age reducing the clinical value of the $\mathrm{D}$-dimer assay in the elderly [5].

Through the age-stratification study, we found that the elderly had higher incidence of thrombosis, which is consistent with the conclusion of our risk factor analysis, and by the age-adjusted D-dimer cut-off value in all age groups, it can be found that more samples are lower than the cut-off value, which improved the sensitivity of the model.

In our study, the age-adjusted $\mathrm{D}$-dimer value was defined as age $\times 0.01(\mu \mathrm{g} / \mathrm{mL})$, which was considered safe. Nybo and Hvas had found that the age-adjusted Ddimer cut-off was safe to recommend the use of an ageadjusted D-dimer in a DVT setting as well as for PE from a systematic review [9]. In this study, we also made multivariate logistic regression analysis to find that age is the risk factor of DVT.

In previous reports, Imai et al. demonstrated that age and D-dimer index can be useful in screening patients for DVTs before THA [13]. The conclusion was similar to us. Moreover, age-adjusted D-dimer value was calculated as patients' age times $0.01(\mu \mathrm{g} / \mathrm{mL})$ by Schouten et al. [10]. They drew conclusions that age-adjusted Ddimer value can increase specificity, whereas sensitivity was not. The sensitivity was $97 \%$, but the specificity was below $60 \%$. The results did not coincide with our research. In addition, some studies focused on the performance of age-adjusted D-dimer cut-off values for reducing the use of ultrasounds $[3,14,15]$. Broen et al. inclined the view that age-adjusted D-dimer cut-off values can lead to less duplex ultrasounds performed to find patients that suffer from DVT [16].

In our study, the age-adjusted D-dimer value cut-off increased sensitivity, which was important to find patients with DVT. DVT was the most critical complication. Once we did not observe the asymptomatic DVT patients, it might cause serious consequences, such as pulmonary embolism (PE). Thus, the ageadjusted D-dimer could help us find more asymptomatic DVT patients. Actually, that was what we cared about more.

As far as we know, it was the first study to focus on the performance of age-adjusted D-dimer cut-off values for the screening of deep vein thrombosis in both TKA and THA. We gave the lower extremity venous color

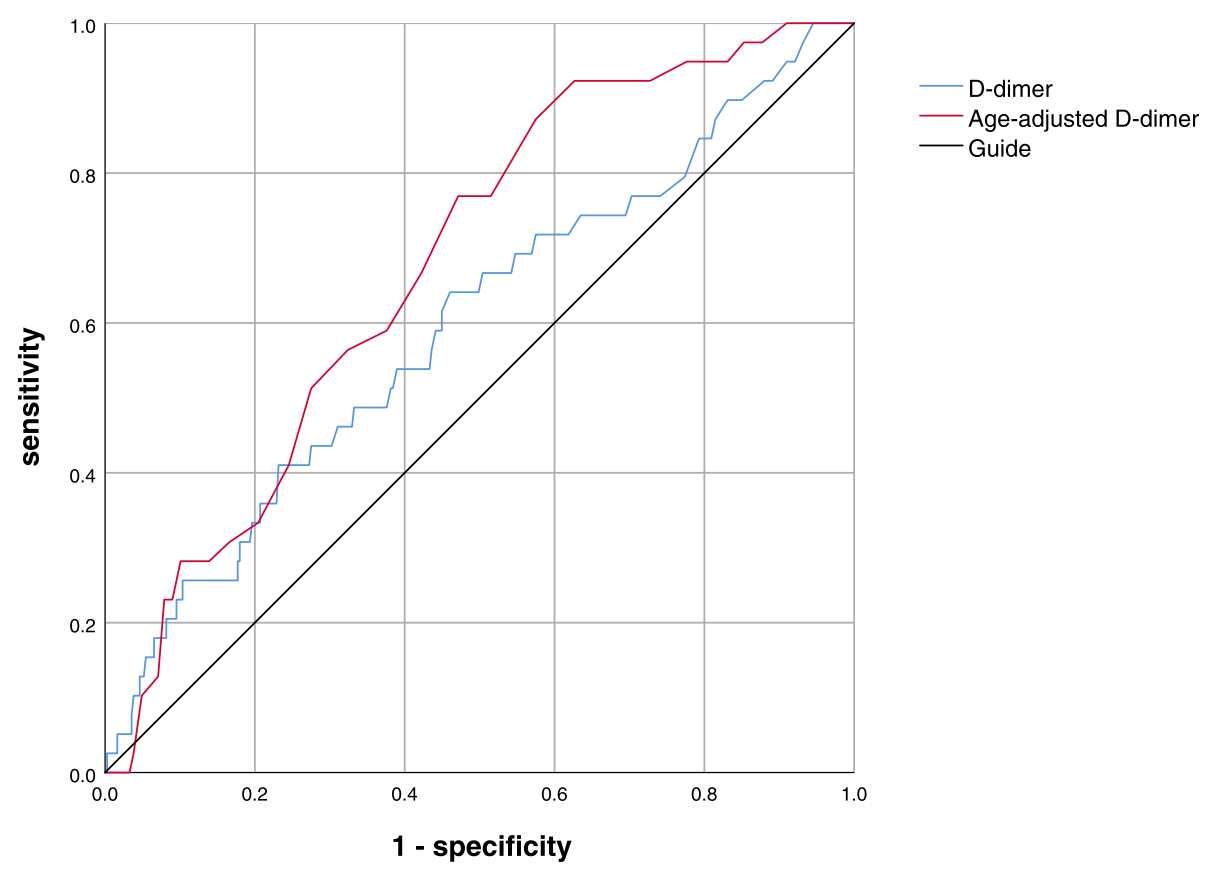

Fig. 1 The ROC curve for the age-adjusted D-dimer and the traditional D-dimer 
Table 4 AUC and cut-off

\begin{tabular}{lllllll}
\hline & Cut-off value & Sensitivity & Specificity & AUC & $\mathbf{9 5 \% ~ C l}$ & $\boldsymbol{p}$ value \\
\hline Age-adjusted D-dimer & 0.655 & 0.769 & 0.768 & 0.795 & $0.611-0.881$ & $<0.001$ \\
D-dimer & 0.845 & 0.41 & 0.658 & 0.685 & $0.499-0.696$ & 0.045 \\
\hline
\end{tabular}

$A \cup C$ area under the curve, $\mathrm{Cl}$ confidence interval

ultrasound bedside to all patients admitted to the hospital. Thus, we had enough samples for observation.

However, there were several limitations in our study. First, we did not use Wills score to assess the sensitivity and specificity of age-adjusted D-dimer cutoff value. Wills score could help us predict DVT better [17]. Second, our hospital used latex agglutination turbidimetry (LATEX) to measure the concentrations of D-dimer. Compared to enzyme-linked fluorescent assays (ELISA), LATEX may lead to lower sensitivity and specificity in our study. Finally, we did not group patients to several levels by age, which may get a better result in this study.

In conclusion, compared to traditional D-dimer, ageadjusted D-dimer showed better performance in screening DVT, which was useful clinically. Meanwhile, more clinical observations were needed to verify our ideas.

\section{Supplementary Information}

The online version contains supplementary material available at https://doi. org/10.1186/s13018-020-02172-w.

Additional file 1. Supplementary Material.

\section{Abbreviations}

DVT: Deep vein thrombosis; TKA: The knee arthroplasty; THA: The hip arthroplasty; TJA: The joint arthroplasty; PTE: Pulmonary thromboembolism; BMI: Body mass index; ROC: Receiver operating characteristic; AUC: Area under the curve; APTT: Activated partial thromboplastin time; PT: Prothrombin time; INR: International normalized ratio; Hb: Hemoglobin; $\mathrm{Cl}$ : Confidence interval; OR: Odds ratio; PE: Pulmonary embolism; ELISA: Enzyme-linked fluorescent assays; LATEX: Latex agglutination turbidimetry

\section{Acknowledgements}

This work was supported by Key Program of NSFC (81730067), Jiangsu Provincial Key Medical Center Foundation, Jiangsu Provincial Medical Outstanding Talent Foundation, Jiangsu Provincial Medical Youth Talent Foundation, Jiangsu Provincial Key Medical Talent Foundation, National Natural Science Foundation of China (81972124), and Basic Research Program of Jiangsu Province (20170123). We thank the patients and their families who donated their blood samples for this study.

\section{Authors' contributions}

Qing Jiang contributed substantially to the conception and design of the study. Jian Xiang Wu, Jiang Hui Qing, Yao Yao, and Dong Yang Chen collected and analyzed the data. Jian Xiang Wu wrote the manuscript with support from Yao Yao. All authors read and approved the final manuscript.

\section{Funding}

This work was supported by Key Program of NSFC (81730067), Jiangsu Provincial Key Medical Center Foundation, Jiangsu Provincial Medical Outstanding Talent Foundation, Jiangsu Provincial Medical Youth Talent
Foundation, Jiangsu Provincial Key Medical Talent Foundation, National Natural Science Foundation of China (81972124), and Basic Research Program of Jiangsu Province (20170123).

\section{Availability of data and materials}

The data used to support the findings of this study are available from the corresponding author upon request.

Ethics approval and consent to participate

This study was approved by the institutional review board (IRB) of Nanjing Drum Tower Hospital (No. 2012029).

\section{Consent for publication}

Not applicable.

\section{Competing interests}

The authors declare that they have no competing interests.

\section{Author details}

${ }^{1}$ Department of Sports Medicine and Adult Reconstructive Surgery, Drum Tower Hospital, School of Nanjing Medical University, 321 Zhongshan Road, Nanjing 210008, Jiangsu, P.R. China. ${ }^{2}$ State Key Laboratory of Pharmaceutical Biotechnology, Department of Sports Medicine and Adult Reconstructive Surgery, Nanjing Drum Tower Hospital, The Affiliated Hospital of Nanjing University Medical School, 321 Zhongshan Road, Nanjing 210008, Jiangsu, P.R. China.

Received: 6 February 2020 Accepted: 25 December 2020

Published online: 25 January 2021

\section{References}

1. Yamaguchi T, et al. Incidence and time course of asymptomatic deep vein thrombosis with fondaparinux in patients undergoing total joint arthroplasty. Thromb Res. 2010;126(4):e323-6.

2. Dai $X$, et al. Associations of serum lipids and deep venous thrombosis risk after total knee arthroplasty in patients with primary knee osteoarthritis. Int J Low Extrem Wounds. 2019:1534734619868123.

3. Sartori M, et al. D-dimer for the diagnosis of upper extremity deep and superficial venous thrombosis. Thromb Res. 2015;135(4):673-8.

4. Ruiz-Gimenez N, et al. Rapid D-dimer test combined a clinical model for deep vein thrombosis. Validation with ultrasonography and clinical followup in 383 patients. Thromb Haemost. 2004;91(6):1237-46.

5. Harper $\mathrm{PL}$, et al. D-dimer concentration increases with age reducing the clinical value of the D-dimer assay in the elderly. Intern Med J. 2007;37(9): 607-13

6. Cini $\mathrm{M}$, et al. D-dimer use for deep venous thrombosis exclusion in elderly patients: a comparative analysis of three different approaches to establish cut-off values for an assay with results expressed in D-dimer units. Int J Lab Hematol. 2014;36(5):541-7.

7. Farm M, et al. Age-adjusted D-dimer cut-off leads to more efficient diagnosis of venous thromboembolism in the emergency department: a comparison of four assays. J Thromb Haemost. 2018;16(5):866-75.

8. Li J, et al. The diagnostic efficacy of age-adjusted D-dimer cutoff value and pretest probability scores for deep venous thrombosis. Clin Appl Thromb Hemost. 2019:25:1076029619826317.

9. Nybo M, Hvas AM. Age-adjusted D-dimer cut-off in the diagnostic strategy for deep vein thrombosis: a systematic review. Scand J Clin Lab Invest. 2017 77(8):568-73.

10. Schouten HJ, et al. Diagnostic accuracy of conventional or age adjusted Ddimer cut-off values in older patients with suspected venous 
thromboembolism: systematic review and meta-analysis. BMJ. 2013;346: f2492.

11. Haenssle HA, et al. Limited diagnostic value of Wells-score and D-dimer testing in hospitalized dermatologic patients with symptoms of deep vein thrombosis. Eur J Dermatol. 2013;23(6):830-6.

12. Fluss R, Faraggi D, Reiser B. Estimation of the Youden Index and its associated cutoff point. Biom J. 2005;474(4):458-72.

13. Imai N, et al. Usefulness of a novel method for the screening of deep vein thrombosis by using a combined D-dimer- and age-based index before total hip arthroplasty. PLoS One. 2017;12(2):e0172849.

14. Riva $\mathrm{N}$, et al. Age-adjusted D-dimer to rule out deep vein thrombosis: findings from the PALLADIO algorithm. J Thromb Haemost. 2018;16(2): 271-8.

15. Prochaska JH, et al. Age-related diagnostic value of D-dimer testing and the role of inflammation in patients with suspected deep vein thrombosis. Sci Rep. 2017;7(1):4591.

16. Broen K, Scholtes B, Vossen R. Predicting the need for further thrombosis diagnostics in suspected DVT is increased by using age adjusted D-dimer values. Thromb Res. 2016;145:107-8.

17. Sartori $\mathrm{M}$, et al. The Wells rule and D-dimer for the diagnosis of isolated distal deep vein thrombosis. J Thromb Haemost. 2012;10(11):2264-9.

\section{Publisher's Note}

Springer Nature remains neutral with regard to jurisdictional claims in published maps and institutional affiliations.

Ready to submit your research? Choose BMC and benefit from:

- fast, convenient online submission

- thorough peer review by experienced researchers in your field

- rapid publication on acceptance

- support for research data, including large and complex data types

- gold Open Access which fosters wider collaboration and increased citations

- maximum visibility for your research: over $100 \mathrm{M}$ website views per year

At $\mathrm{BMC}$, research is always in progress.

Learn more biomedcentral.com/submissions 